\title{
A Max-SAT Solver with Lazy Data Structures
}

\author{
Teresa Alsinet, Felip Manyà, and Jordi Planes \\ Departament d'Informàtica \\ Universitat de Lleida,Jaume II, 69, 25001-Lleida, Spain \\ \{racy,felip,jordi\}@eup.udl.es
}

\begin{abstract}
We present a new branch and bound algorithm for Max-SAT which incorporates original lazy data structures, a new variable selection heuristics and a lower bound of better quality. We provide experimental evidence that our solver outperforms some of the best performing MaxSAT solvers on a wide range of instances.
\end{abstract}

Keywords: Max-SAT, branch and bound, lower bound, heuristics, data structure.

\section{Introduction}

In recent years we have seen an increasing interest in propositional satisfiability (SAT) that has led to the development of fast and sophisticated complete SAT solvers like Chaff, Grasp, RelSat and Satz, which are based on the well-known Davis-Putnam-Logemann-Loveland (DPLL) procedure [5]. Given a Boolean CNF formula $\phi$, such algorithms determine whether there is a truth assignment that satisfies $\phi$. Unfortunately, they are not able to solve a well-known satisfiability optimization problems: Max-SAT. Given a Boolean CNF formula $\phi$, Max-SAT consists of finding a truth assignment that maximizes the number of satisfied clauses in $\phi$. When all the clauses have at most $k$ literals per clause Max-SAT is called Max- $k$-SAT.

To our best knowledge, there are only three exact algorithms for Max-SAT that are variants of the DPLL procedure. The first was developed by Wallace \& Freuder [15] (WF), the second was developed by Borchers \& Furman [3] $(\mathrm{BF})$, and the third, which is based on $\mathrm{BF}$, was developed by Alsinet, Manyà \& Planes [2] (AMP). All of them are depth-first branch and bound algorithms. The first was implemented in Lisp, while the rest were implemented in $\mathrm{C}$ and are publicly available. There are other exact algorithms for Max-SAT, but based on mathematical programming techniques $[4,6,8]$. There are also two exact DPLL-based algorithms for solving Max-2-SAT: one is due to Zhang, Shen \& Manyà [16] (ZSM), and the other to Alber, Gramm \& Niedermeier [1] (AGN).

In this paper we first present a new branch and bound algorithm for MaxSAT which incorporates original lazy data structures, a new variable selection heuristics, and a lower bound of better quality. We then report on an experimental investigation we have conducted in order to evaluate our solver on Max-SAT 
instances. The results obtained provide experimental evidence that our solver outperforms some of the best performing existing Max-SAT solvers on a wide range of instances.

Our new Max-SAT solver, which we call Lazy, differs from BF and AMP in the data structures used to represent and manipulate CNF formulas, in the lower bound, in the variable selection heuristic and in the incorporation of the Dominating Unit Clause (DUC) rule.

\section{Branch and Bound for Max-SAT}

The space of all possible assignments for a CNF formula $\phi$ can be represented as a search tree, where internal nodes represent partial assignments and leaf nodes represent complete assignments. A branch and bound algorithm for Max-SAT explores the search tree in a depth-first manner. At every node, the algorithm compares the number of clauses unsatisfied by the best complete assignment found so far - called upper bound $(U B)$ - with the number of clauses unsatisfied by the current partial assignment (unsat) plus an underestimation of the number of clauses that become unsatisfied if we extend the current partial assignment into a complete assignment (underestimation). The sum unsat + underestimation is called lower bound $(L B)$. Obviously, if $U B \leq L B$, a better assignment cannot be found from this point in search. In that case, the algorithm prunes the subtree below the current node and backtracks to a higher level in the search tree. If $U B>L B$, it extends the current partial assignment by instantiating one more variable; which leads to create two branches from the current branch: the left branch corresponds to instantiate the new variable to false, and the right branch corresponds to instantiate the new variable to true. In that case, the formula associated with the left (right) branch is obtained from the formula of the current node by deleting all the clauses containing the literal $\neg p(p)$ and removing all the occurrences of the literal $p(\neg p)$; i.e., the algorithm applies the one-literal rule [9]. The solution to Max-SAT is the value that $U B$ takes after exploring the entire search tree.

Borchers \& Furman [3] designed and implemented a branch and bound solver for Max-SAT, called BF herein, that incorporates two quite significant improvements:

- Before starting to explore the search tree, they obtain an upper bound on the number of unsatisfied clauses in an optimal solution using the local search procedure GSAT [13].

- When branching is done, branch and bound algorithms for Max-SAT apply the one-literal rule (simplifying with the branching literal) instead of applying unit propagation as in the DPLL-style solvers for SAT. ${ }^{1}$ If unit propagation is applied at each node, the algorithm can return a non-optimal

\footnotetext{
${ }^{1}$ By unit propagation we mean the repeated application of the one-literal rule until a saturation state is reached.
} 
solution. However, when the difference between the lower bound and the upper bound is one, unit propagation can be safely applied, because otherwise by fixing to false any literal of any unit clause we reach the upper bound. Borchers \& Furman perform unit propagation in that case.

The improvements incorporated in $\mathrm{BF}$ are also used in the rest of DPLLbased Max-SAT solvers considered in this paper (i.e. AMP, ZSM, AGN).

The lower bound and variable selection heuristic of $\mathrm{BF}$ are:

$-\mathrm{LB}_{\mathrm{BF}}=$ unsat. Note that that the number of clauses unsatisfied by the current partial assignment coincides with the number of empty clauses that contains the formula associated with the current partial assignment. In this elementary lower bound there is no underestimation of the number of clauses that become unsatisfied if we extend the current partial assignment into a complete assignment.

- MOMS: selects a variable among those that appear more often in clauses of minimum size. That is the heuristic of Borchers \& Furman. Ties are broken by choosing the first variable in lexicographical order [12].

In a previous paper [2], we incorporated two improvements into BF that led to significant performance improvements: a lower bound of better quality $\left(\mathrm{LB}_{\mathrm{AMP}}\right)$ and another variable selection heuristic $(\mathrm{JW})$ :

$-\mathrm{LB}_{\mathrm{AMP}}=$ unsat $+\sum_{p \in \phi^{\prime}} \min (i c(p), i c(\neg p))$, where $\phi^{\prime}$ is the formula associated with the current partial assignment, and $i c(p)(i c(\neg p))$-inconsistency count of $p(\neg p)$ - is the number of clauses that become unsatisfied if the current partial assignment is extended by fixing $p$ to true (false). Note that $i c(p)(i c(\neg p))$ coincides with the number of unit clauses of $\phi^{\prime}$ that contain $\neg p(p)$.

If for each variable we count the number of positive and negative literals in unit clauses, we can know the number of unit clauses that will not be satisfied if the variable is instantiated to true or false. Obviously, the total number of unsatisfied clauses resulting from either instantiation of the variable must be greater than or equal to the minimum count. Moreover, the counts for different variables are independent, since they refer to different unit clauses. Hence, by summing the minimum count for all variables in unit clauses and adding this sum to the number of empty clauses, we calculate a lower bound for the number of unsatisfied clauses given the current assignment. Such a lower bound was considered in [15].

- Jeroslow-Wang (JW) [7]: given a formula $\phi$, for each literal $L$ of $\phi$ the following function is defined: $J(L)=\sum_{L \in C \in \phi} 2^{-|C|}$, where $|C|$ is the length of clause $C$. JW selects a variable $p$ of $\phi$ among those that maximize $J(p)+J(\neg p)$.

Our solver AMP is basically BF with the above lower bound and variable selection heuristic. Figure 1 shows the pseudo-code of the skeleton of BF and AMP. We use the following notation: empty-clauses $(\phi)$ is a function that returns the number of empty clauses in $\phi$; lower-bound $(\phi)$ is the sum of the 
Input: $\max -\operatorname{sat}(\phi, u b)$ : A Boolean CNF formula $\phi$ and an upper bound $u b$

1: if $\phi=\emptyset$ or $\phi$ only contains empty clauses then

2: $\quad$ return empty-clauses $(\phi)$

3: end if

4: if lower-bound $(\phi) \geq u b$ then

5: $\quad$ return $\infty$

6: end if

7: if unsat $=u b-1$ then

8: $\phi \leftarrow$ unit-propagation $(\phi)$

9: end if

10: $p \leftarrow$ select-variable $(\phi)$

11: $u b \leftarrow \min \left(u b, \max -\operatorname{sat}\left(\phi_{\neg p}, u b\right)\right)$

12: return $\min \left(u b, \max -\operatorname{sat}\left(\phi_{p}, u b\right)\right)$

Output: The minimum number of clauses of $\phi$ that can be unsatisfied

Fig. 1. Branch and Bound for Max-SAT

number of empty clauses in $\phi$ plus an underestimation of the number of unsatisfied clauses in the formula obtained from $\phi$ by removing its empty clauses. In our case, $\mathrm{LB}_{\mathrm{BF}}$ or $\mathrm{LB}_{\mathrm{AMP}} ; u b$ is an upper bound of the number of unsatisfied clauses in an optimal solution. We assume that the input value is that obtained with GSAT; select-variable $(\phi)$ is a function that returns a variable of $\phi$ following an heuristic; in our case, MOMS or JW; and $\phi_{p}\left(\phi_{\neg p}\right)$ is the formula obtained by applying the one-literal rule to $\phi$ using the literal $p(\neg p)$.

\subsection{A new Max-SAT solver}

Our new Max-SAT solver, which we call Lazy, differs from previous solvers in the data structures used to represent and manipulate CNF formulas, in the lower bound, in a novel variable selection heuristic, in the preprocessing of such formulas and in the incorporation of the Dominating Unit Clause rule. Lazy was implemented in $\mathrm{C}++$.

Data structures BF and AMP use adjacency lists to represent CNF formulas and their variable ordering are dynamic. Lazy uses a static variable selection heuristic (defined below) that allows us to implement extremely efficient data structures for representing and manipulating CNF formulas. Our data structures take into account the following fact: we are only interested in knowing when a clause has become unit or empty. Thus, if we have a clause with four variables, we do not perform any operation in that clause until three of the variables appearing in the clause have been instantiated; i.e., we delay the evaluation of a clause with $k$ variables until $k-1$ variables have been instantiated. In our case, as we instantiate the variables using a static order, we do not have to evaluate a clause until the penultimate variable of the clause in the static order has been instantiated. 
The data structures are defined as follows: For each clause we have a pointer to the penultimate variable of the clause in the static order, and the clauses of a CNF formula are ordered by that pointer. We also have a pointer to the last variable of the clause. When a variable $p$ is fixed to true (false), only the clauses whose penultimate variable in the static order is $\neg p(p)$ are evaluated. This approach has two advantages: the cost of backtracking is constant (we do not have to undo pointers like in adjacency lists) and, at each step, we evaluate a minimum number of clauses.

For instance, suppose we have a formula with the following clauses and that variables are instantiated in lexicographic order:

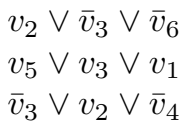

If $v_{1}$ and $v_{2}$ have been instantiated to false, when we branch on $v_{3}=$ true we derive the unit clauses $\bar{v}_{6}$ and $\bar{v}_{4}$; and when we branch on $v_{3}=$ false we derive the unit clause $v_{5}$. The data structure snapshot is shown in Fig. 2.

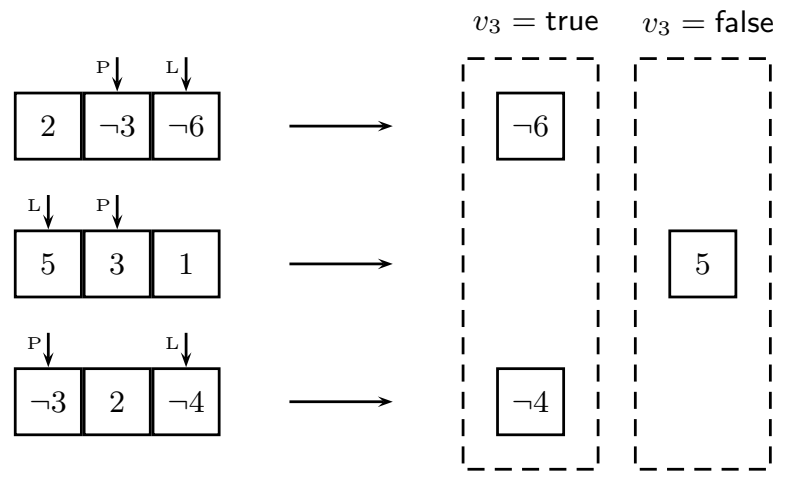

Fig. 2. Lazy data structure snapshot example. The numbers in the boxes stand for the literals with the given variable number. The arrow $L$ stands for the Last pointer and the arrow $P$ stands for the Penultimate pointer.

Lower bound Lazy incorporates a lower bound ( $\left.\mathrm{LB}_{\mathrm{LAZY}}\right)$ of better quality than $\mathrm{LB}_{\mathrm{BF}}$ and $\mathrm{LB}_{\mathrm{AMP}}$. $\mathrm{LB}_{\mathrm{LAZY}}$ can be understood as $\mathrm{LB}_{\mathrm{AMP}}$ extended with a specialization of the so-called star rule defined in [11]. The star rule states that if we have a clause of the form $p_{1} \vee \cdots \vee p_{k}$ and $k$ unit clauses of the form $\neg p_{1}, \ldots, \neg p_{k}$, then the lower bound can be incremented by one. In our case, we only consider clauses of length two. For longer clauses the star rule does not lead to performance improvements. The pseudo-code of $\mathrm{LB}_{\mathrm{LAZY}}$ is defined as follows [14]: 


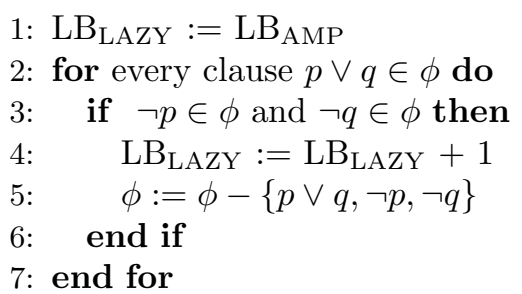

Variable selection heuristic MOMS is a branching heuristic that selects a variable among those that appear more often in clauses of minimum size, and breaks ties by choosing the first variable in lexicographical order. We have defined MOMS*, which is like MOMS but breaks ties in a different way. In MOMS* ties are broken by choosing the variable $x$ with the highest weight $w$. Such a weight is calculated by the product $w(x)=\prod_{l \in N} \mathrm{oc}(l)$, where $N$ stands for the set of negated neighbouring literals of variable $x$, and oc $(l)$ stands for the number of ocurrences of literal $l$. The reason behind the calculation is that any clause $a \vee b \vee c$ can be seen as the implication $\neg a \wedge \neg b \rightarrow c$. So, the bigger the product oc $(\neg a) \cdot \operatorname{oc}(\neg b)$, the higher the probability of creating the unit clause $c$.

Note that MOMS is used as a dynamic variable selection heuristic in BF while MOMS* is used as a static variable selection heuristic in Lazy.

Formula reduction preprocessing Before the search starts, the initial formula is simplified by applying the resolution rule to some binary clauses. For every pair of clauses $a \vee b$ and $\neg a \vee b$ such that variable $a$ precedes variable $b$ in the static instantiation order, Lazy reduces them to the unit clause $b$.

Dominating Unit Clause (DUC) rule DUC is an inference rule, defined in [11], that allows us to fix the truth value of a variable; i.e., it avoids to apply branching on that variable. DUC is defined as follows: If a CNF formula $\phi$ has $k$ occurrences of a variable $p(\neg p)$ and has at least $k$ unit clauses of the form $\neg p$ $(p)$, then the value of $p$ can be set to false (true).

\section{Experimental Results}

We conducted an experimental investigation in order to compare the performance of BF, AMP, and Lazy. When dealing with Max-2-SAT instances, we also compare with ZSM and AGN. The experiments were performed on a $2 \mathrm{GHz}$ Pentium IV with $512 \mathrm{Mb}$ of RAM under Linux.

In our first experiment, we evaluated the relevance of defining lazy data structures to get substantial performance improvements. To this end, we compared BF and Lazy using a simple variable selection heuristic: variables are instantiated in lexicographical order. Moreover, we removed all the improvements we introduced into Lazy and replaced $\mathrm{LB}_{\mathrm{LAZY}}$ with $\mathrm{LB}_{\mathrm{BF}}$. In this way, we have that BF and Lazy traverse the same search tree. Figure 3 shows the results obtained when solving sets of randomly generated Max-3-SAT instances with 30 
variables and a different number of clauses. Such instances were generated using the method described in [10]. We generated sets for 180, 240, 300, 360, 420 and 480 clauses, where each set had 100 instances. We observe that this modified version of Lazy is about 5 times faster than BF when both solvers traverse the same search tree.

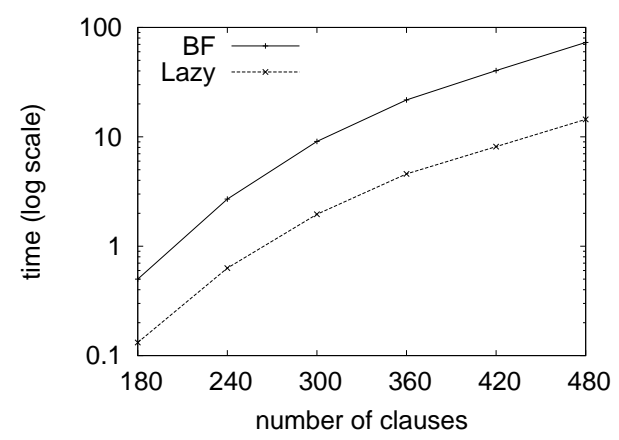

Fig. 3. Experimental results for 30-variable Max-3-SAT instances. Mean time (in seconds).
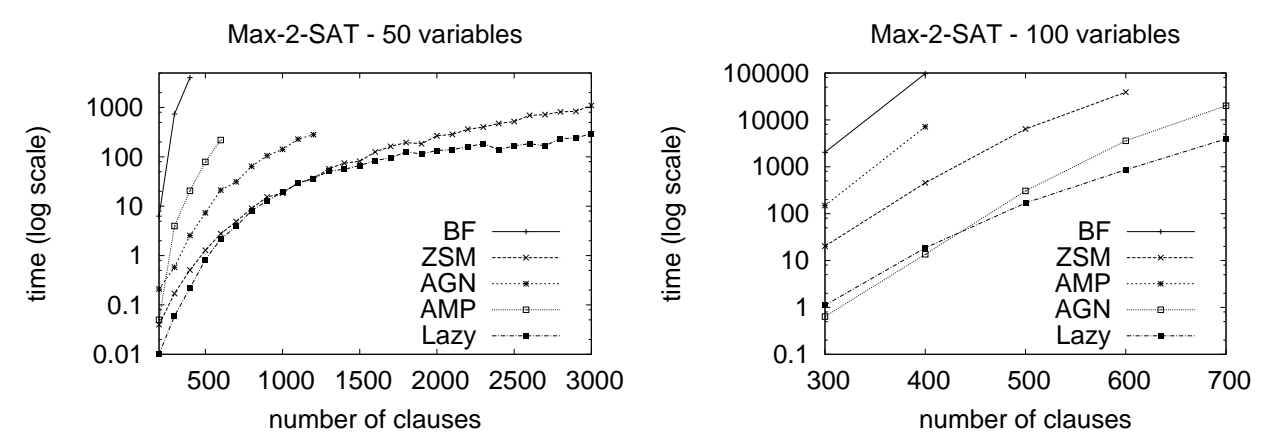

Fig. 4. Experimental results for 50-variable and 100-variable Max-2-SAT instances. Mean time (in seconds).

In our second experiment, we generated sets of random Max-2-SAT instances with 50 and 100 variables and a different number of clauses. Such instances were generated using the method described in [10], and each set had 100 instances. The results of solving such instances with BF, AMP, ZSM, AGN and Lazy are shown in Fig. 4. Along the horizontal axis is the number of clauses, and along 
the vertical axis is the mean and median time (in seconds) needed to solve an instance of a set. Notice that we use a log scale to represent run-time. Clearly, Lazy outperforms the rest of solvers, even ZSM and AGN that are specifically designed to solve Max-2-SAT instances.
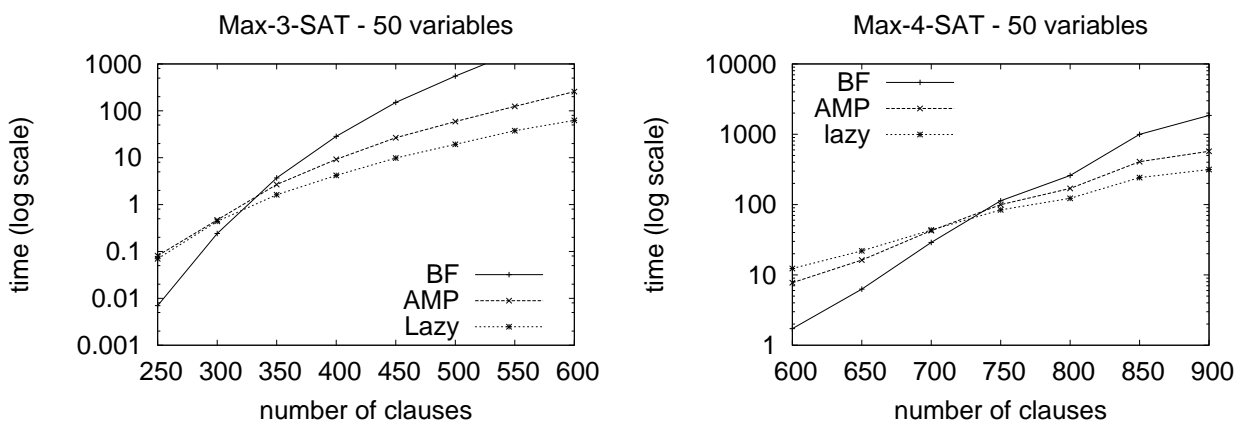

Fig. 5. Experimental results for 50-variable Max-3-SAT (left) and Max-4-SAT (right) instances. Mean time (in seconds).

In our third experiment, we generated sets of random Max-3-SAT and Max4-SAT instances with 50 variables and a different number of clauses. Such instances were generated using the method described in [10], and each set had 100 instances. The results of solving such instances with BF, AMP, and Lazy are shown in Fig. 5. Again, we clearly see that Lazy provides substantial performance improvements, which are due to the lazy data structures, the quality of the lower bound and the variable selection heuristic used.

Acknowledgements Research partially supported by project CICYT TIC2001-1577-C03-03 funded by the Ministerio de Ciencia y Tecnología.

\section{References}

1. J. Alber, J. Gramm, and R. Niedermeier Faster exact algorithms for hard problems: A parameterized point of view In 25th Conf. on Current Trends in Theory and Practice of Informatics, LNCS, pages 168-185. Springer-Verlag, 1998.

2. T. Alsinet, F. Manyà, and J. Planes Improved branch and bound algorithms for Max-SAT In Proceedings of the 6th International Conference on the Theory and Applications of Satisfiability Testing, 2003.

3. B. Borchers and J. Furman A two-phase exact algorithm for MAX-SAT and weighted MAX-SAT problems J. of Combinatorial Optimization, 2:299-306, 1999.

4. J. Cheriyan, W. Cunningham, L. Tunçel, and Y. Wang A linear programming and rounding approach to MAX-2-SAT In D. Johnson and M. Trick, editors, Cliques, Coloring and Satisfiability, volume 26, pages 395-414. 1996. 
5. M. Davis, G. Logemann, and D. Loveland A machine program for theorem-proving Communications of the ACM, 5:394-397, 1962.

6. E. de Klerk and J. P. Warners Semidefinite programming approaches for MAX2-SAT and MAX-3-SAT: computational perspectives Technical report, Delft, The Netherlands, 1998

7. R. G. Jeroslow and J. Wang Solving propositional satisfiability problems Annals of Mathematics and Artificial Intelligence, 1:167-187, 1990.

8. S. Joy, J. Mitchell, and B. Borchers A branch and cut algorithm for max-sat and weighted max-sat In DIMACS Workshop on Satisfiability: Theory and Applications, 1996.

9. D. W. Loveland Automated Theorem Proving. A Logical Basis, volume 6 of Fundamental Studies in Computer Science North-Holland, 1978.

10. D. Mitchell, B. Selman, and H. Levesque Hard and easy distributions of SAT problems In Proceedings of AAAI'92, pages 459-465, 1992.

11. R. Niedermeier and P. Rossmanith New upper bounds for maximum satisfiability Journal of Algorithms, 36:63-88, 2000.

12. D. Pretolani Efficiency and stability of hypergraph SAT algorithms In Proceedings of the DIMACS Challenge II Workshop, 1993.

13. B. Selman, H. Levesque, and D. Mitchell A new method for solving hard satisfiability problems In Proceedings of AAAI'92, pages 440-446, 1992.

14. Shen, H., Zhang, H. Improving Exact Algorithms for MAX-2-SAT In Proceedings of the 8th International Symposium on Artificial Intelligence and Mathematics, Fort Lauderdale, Florida, 2004.

15. R. Wallace and E. Freuder Comparative studies of constraint satisfaction and Davis-Putnam algorithms for maximum satisfiability problems In D. Johnson and M. Trick, editors, Cliques, Coloring and Satisfiability, volume 26, pages 587-615. 1996.

16. H. Zhang, H. Shen, and F. Manyà Exact algorithms for MAX-SAT In Proceedings of the 4th International Workshop on First-Order Theorem Proving, FTP-2003, Valencia, Spain, 2003. 